\title{
Confirmación de la presencia de Clerodendrum bungei (Lamiaceae) en la Argentina
}

\author{
Confirmation of the presence of Clerodendrum bungei (Lamiaceae) in Argentina
}

\author{
Adela M. Panizza ${ }^{1,2}$ (D) \& Christian A. Zanotti ${ }^{3}$ (i)
}

\begin{abstract}
Resumen: Clerodendrum bungei Steud. es confirmada para la Flora Argentina, citada anteriormente sin material de referencia. Se presenta una breve descripción de la especie, fotos de campo y comentarios sobre su distribución geográfica y ecología. Además, se provee una clave de las especies adventicias y nativas de Clerodendrum en Argentina.
\end{abstract}

Palabras clave: Argentina, Clerodendrum, especie exótica, flora, Lamiaceae.

Summary: Clerodendrum bungei Steud. is confirmed for the Argentinean Flora, cited before without reference material. A brief description of the species, field photos and comments on its geographical distribution and ecology are presented. In addition, a key to the adventitious and native species of Clerodendrum in Argentina is provided.

Key words: Argentina, Clerodendrum, exotic species, flora, Lamiaceae.

\section{Introducción}

Clerodendrum L. pertenece actualmente a la familia Lamiaceae (Cantino, 1992; Cantino et al., 1992), es un género morfológicamente heterogéneo y se reconstruye como polifilético (Steane et al., 2004; Yuan et al., 2010; Wearn et Mabberley, 2011a; 2011b). La taxonomía de este género es y ha sido controversial, debido a la amplia descripción de variedades ornamentales, la existencia de hibridación y la extrema variabilidad causada por factores ambientales y por la selección de cultivares (Rueda, 1993). Steane et al. (2004) y Yuan et al. (2010) redefinen a Clerodendrum en base a estudios taxonómicos moleculares y una reevaluación de los caracteres morfológicos, limitando a los taxones de este género exclusivamente a África y Asia. Teniendo en cuenta esta hipótesis, los autores transfieren algunas de las especies y rehabilitan géneros netamente americanos anteriormente fundados: Aegiphila Jacq., Amasonia L. f. y Tetraclea A. Gray, para las especies neotropicales de Clerodendrum. Sin embargo, Yuan et al. (2010) no efectúa la totalidad de las combinaciones de las especies neotropicales del género, por lo que actualmente algunos taxones aún se encuentran bajo Clerodendrum L., como por ejemplo C. ekmanii Moldenke, especie nativa de la Argentina, Brasil y Paraguay (Buchoski, 2021; Zuloaga et al., 2019).

\footnotetext{
${ }^{1}$ Programa de Pós Graduação de Mestrado em Biodiversidade Neotropical (PPBN), Herbario Evaldo Buttura, Universidad Federal de Integración Latino-Americana (EVB-UNILA), Foz do Iguaçu, Paraná, Brasil. e-mail: adela. maria.panizza@gmail.com

${ }^{2}$ Facultad de Ciencias Forestales - Universidad Nacional de Misiones (FacFor - UNaM), Eldorado, Misiones, Argentina.

${ }^{3}$ Instituto de Botánica Darwinion (IBODA-CONICET), San Isidro, Buenos Aires, Argentina.
} 
Este género afroasiático, según Yuan et al. (2010), contiene cerca de 150 especies que habitan generalmente en bordes de selvas y bosques ribereños (Wearn et Mabberley, 2011a; 2011b). Tiene importancia ecológica, ya que algunas especies son colonizadoras en etapas sucesionales tempranas en tierras degradadas $\mathrm{y}$, además, poseen complejas interacciones con microfungi, hormigas y polinizadores (Wearn et Mabberley, 2011a; $2011 b)$. Se caracterizan por ser árboles, arbustos, lianas o hierbas perennes, de hojas opuestas o verticiladas, con inflorescencias en cimas laxas o formando densas cabezuelas o racimos; las flores presentan el tubo corolino estrechamente cilíndrico y generalmente sobrepasa varias veces la longitud del cáliz (Troncoso, 1974).

Es un género frecuentemente cultivado y por esta razón, se encuentra naturalizado en todo el mundo (Woodson et al., 1973; Troncoso, 1974; Rueda, 1993; Waterhouse, 1993; Idárraga-Piedrahita et al., 2011). En los primeros trabajos sobre el género en la Argentina, se ha citado una especie nativa para Misiones, Clerodendrum ekmanii Moldenke (Moldenke, 1940) y dos especies introducidas, Clerodendrum chinense (Osbeck) Mabb. (sub. Clerodendron fragrans Vent. var. pleniflora Schauer), registrada para Córdoba (Moldenke, 1948) y Tucumán (Troncoso, 1974); y Clerodendrum bungei Steud, para Catamarca (Moldenke, 1948). Sin embargo, las citas de Moldenke no incluyen ejemplares de referencia, y por lo tanto no es posible constatar la procedencia de las mismas. Por otro lado, O'Leary (2018), afirma que $C$. chinense, citada para Salta y Tucumán por Zuloaga et al. (2008, 2019), se basaron sobre material cultivado, a excepción de la colección Venturi 286 (SI) procedente de Tucumán, y por este motivo, cita a esta especie como de presencia dudosa para la Flora Argentina.

A causa de colecciones recientes y de la observación de material de herbario, se ha permitido dar cuenta de la naturalización en la Argentina de Clerodendrum bungei, lo cual se documenta en la presente contribución, confirmando así, la cita de Moldenke (1948) para esta especie. La especie es descripta, ilustrada mediante fotografías y, además, se brinda una clave y fotografías de campo para diferenciar a las tres especies que habitan en la Argentina.

\section{Materiales y Métodos}

El hallazgo y confirmación de la presencia de Clerodendrum bungei en la Argentina, se fundamenta sobre la base de dos ejemplares, uno coleccionado en el 2018 en la provincia de Jujuy (Argentina), y otro en el año 1918 en la provincia de Tucumán (Argentina), ambos depositados en SI (Thiers, 2021). Además, se consultó la bibliografía específica del género (Woodson et al., 1973; Troncoso, 1974; Rueda, 1993; Wearn et Mabberley, 2011b; O’Leary, 2018).

\section{Resultados}

Clerodendrum bungei Steud., Nomencl. Bot. (ed. 2) 1: 382. 1840. Volkameria bungei (Steud.) Lavallée, Énum. Arbres: 179. 1877. Clerodendrum foetidum Bunge, Enum. Pl. China Bor: 52. 1833, nom. illeg., non D. Don, 1825. Typus: China, Bunge 296 (Holotypus, probablemente LE, no localizado). Fig. 1 A-D

Arbusto de 1-2(-3) $\mathrm{m}$ de alto, caducifolio, con ramificaciones teretes, subteretes a tetrágonas, lenticeladas, pubescentes. Hojas opuestas, decusadas, con pecíolos de 2-4(14) cm long., glabros a glabrescentes; láminas ovada-deltoideas a elípticas, de 8-20 $\times 3-15 \mathrm{~cm}$, de ápice acuminado a agudo, base generalmente cuneada, truncada o cordada, a veces ligeramente decurrente, con el margen aserrado-dentado, papirácea, glabra o con tricomas glandulares en ambas caras. Inflorescencias corimbosas-paniculadas, terminales, densas, de 4-9 × 3-15 cm, pedúnculos ausentes o hasta de $5 \mathrm{~cm}$ long.; brácteas lanceoladas a ovado-lanceoladas, ciliadas, caducas; bractéolas lanceoladas, ciliadas; pedicelos florales de 1-6 mm long., pubescentes. Flores con cáliz tubular o campanulado, de 3-5 mm long., 5-lobado, 

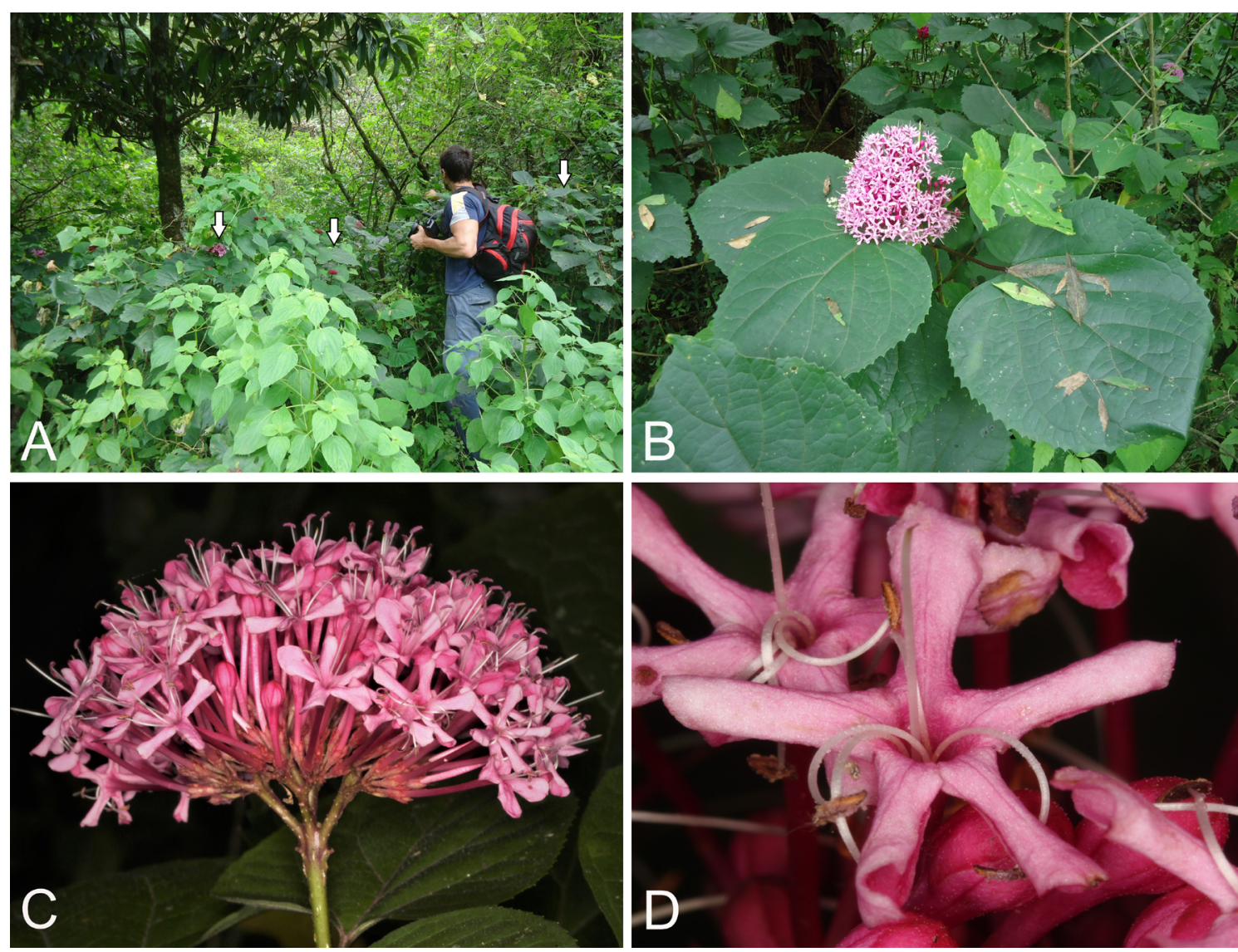

Fig. 1. Clerodendrum bungei. A: Hábito. B: Aspecto general de la inflorescencia y hojas. C: Detalle de la inflorescencia. D: Detalle de la flor (A-D Zanotti \& Panizza 909, créditos fotográficos: A-B: A. M. Panizza; C-D: C. A. Zanotti).

Fig. 1. Clerodendrum bungei. A: Habit. B: General appearance of the inflorescence and leaves. C: Detail of the inflorescence. D: Detail of the flower (A-D Zanotti \& Panizza 909, photo credits: A-B: A. M. Panizza; C-D: C. A. Zanotti).

con los lóbulos ovados, de ápice agudo o acuminado, con glándulas peltadas y tricomas glandulares en su superficie; corola hipocrateriforme, con el tubo de 1-2,5 cm long., 5-lobada, con los lóbulos oblongoovados, de 4-7 mm long., rosada a rojiza o rosado-violácea, glabra; estambres 4 , exertos a 1-1,5 cm de la corola; ovario globoso, estilo de $2-4 \mathrm{~cm}$ long., exerto, más corto o de igual largo que los filamentos estaminales. Fruto no visto.

Fenología: Según los ejemplares observados para la Argentina, florece de diciembre a febrero, pero probablemente su período de floración sea más largo.
Nombres vernáculos: "Bocamelia", "brocamella", "hortensia" (Rueda, 1993; Dimitri, 1980).

Usos: Ornamental y como medicinal, se consume toda la planta en forma de té para la debilidad pulmonar y la tos (Dimitri, 1980).

Distribución geográfica y ecologia: Especie asiática, se distribuye desde China hasta el Norte de la India, entre los 1100-2500 m. Es cultivada y naturalizada intencional e involuntariamente tanto por su valor ornamental y medicinal, en regiones tropicales y subtropicales de todo 
el mundo (Rueda, 1993; Invasive Species Compendium, 2021). En el neotrópico, ha sido citada para México (Rueda, 1993), para el sur y sudeste de Brasil (Harley et al., 2015; Zuloaga et al., 2019), para las Yungas de Bolivia, entre los 1000-1500 m (Jørgensen et al., 2013) y, por último, para la Argentina, ha sido citada para la provincia de Catamarca, sin indicar material de referencia (Moldenke, 1948). La población recientemente hallada, se encontró creciendo espontáneamente en el suroeste de la provincia de Jujuy, cerca de la bifurcación del Río Morado, en el sotobosque de selva ribereña secundaria antropizada en los alrededores de un sendero que conduce al Abra de las Cañas, en el cual el poblado más cercano es la localidad "La Almona", que se encuentra a casi $9 \mathrm{~km}$ del sitio de colección. Por otro lado, también se confirma la presencia de la especie en base a un ejemplar de Venturi 14 (SI), proveniente de Tucumán, en cuya etiqueta de herbario se lee: "terrenos incultos", que según la RAE (2021) significa "terreno que no tiene cultivo ni labor". Las observaciones de la nueva población aquí expuestas, la confirmación del ejemplar procedente de Tucumán y la cita para las Yungas de Bolivia, podrían dar un indicio de que la especie se halla en un proceso de naturalización en esta región, ya que los individuos se reproducen y conforman poblaciones autosustentables, con varios ciclos de vida, y se expanden (por vía sexual o vegetativa) sin la intervención humana (Hurrell et Delucchi, 2013). Esta especie prefiere suelos bien drenados y húmedos, pero tolera la mayoría de los tipos de suelos, como los arenosos y los arcillosos, con un $\mathrm{pH}$ ácido, neutro y alcalino (Rueda, 1993; Jarrett, 2003; PFAF, 2021). Por otro lado, también tolera una temperatura mínima de $15{ }^{\circ} \mathrm{C}$ (Armitage, 2001), sol pleno a sombra parcial, y la sequía (MOBOT, 2021). Se reproduce tanto por semillas como por chupones de raíces, lo que le permite extenderse amplia y rápidamente, formando colonias $\mathrm{y}$, además, es dispersada por humanos y aves (Invasive Species Compendium, 2021). Por otro lado, Nesom (2009) indica que esta especie no se comporta como invasora en el estado de Texas (Estados Unidos de América), y la coloca dentro de una categoría que describe como "Árboles, arbustos, subarbustos y enredaderas leñosas; relativamente poco numerosas, conocidas en relativamente pocas localidades, generalmente en hábitats perturbados, repetidamente introducidos, o quizás simplemente persistiendo en algunas localidades, sin mostrar tendencias agresivamente invasivas".

Material estudiado: ARGENTINA. Jujuy: Dep. San Antonio, subida al Nevado del Castillo por el Hormiguero hasta el Abra de las Cañas, 2419'36"S, 65²6’46”W, $1800 \mathrm{~m}, 20-\mathrm{II}-2018$, fl, Zanotti \& Panizza 909 (SI). Tucumán: Dep. Capital, Villa Luján, 460 m, XII-1918, "terrenos incultos", fl, Venturi 14 (SI).

\section{Clave para diferenciar las especies de Clerodendrum introducidas y nativas de la Argentina}

1. Sufrútices, con los tallos densamente híspidos. Hojas ovadas, de 2-4,5 cm long., de borde crenadoserrado. Inflorescencias no capituliformes, axilares, paucifloras, laxas.

Clerodendrum ekmanii

1'. Arbustos, con los tallos glabros a pubérulos. Hojas deltoides a rómbicas, de 15-30 cm long., de borde sinuado-dentado. Inflorescencias capituliformes, terminales, plurifloras, densas.

2

2. Flor blanca; estambres modificados en pétalos supernumerarios. Clerodendrum chinense

2 '. Flor rosada a rosado-violácea; estambres no modificados en pétalos supernumerarios. 
Clerodendrum chinense (Osbeck) Mabb., The Plant-Book: A Portable Dictionary of the Higher Plants 707. 1989. Fig. 2A-C (Fotos fuera del área de estudio a modo ilustrativo).

Distribución geográfica yecología: En el sur de China, Tailandia, península de Malasia, Sumatra, Java, Borneo, Sulawesi, Molucas y Filipinas; se ha cultivado ampliamente en diversos lugares por lo que su distribución natural en el este de Asia no es clara (Wearn et Mabberley, 2011b). Especie muy cultivada en todas las regiones tropicales y subtropicales del mundo y que tiende a escapar rápidamente de cultivo, en tierras cultivadas abandonadas, bordes de camino y terrenos baldíos en general (Woodson et al., 1973; Wearn et Mabberley, 2011b). Introducida desde el sur de Estados Unidos de América y México, Caribe y Centroamérica hasta la Argentina, Paraguay y Chile (Woodson et al., 1973; Flora Argentina, 2021).

O’Leary (2018: 197) indica que la cita de esta especie para la Flora Argentina, proviene de material de herbario cultivado. Sin embargo, los ejemplares examinados para este trabajo, no presentan ninguna inscripción en su etiqueta

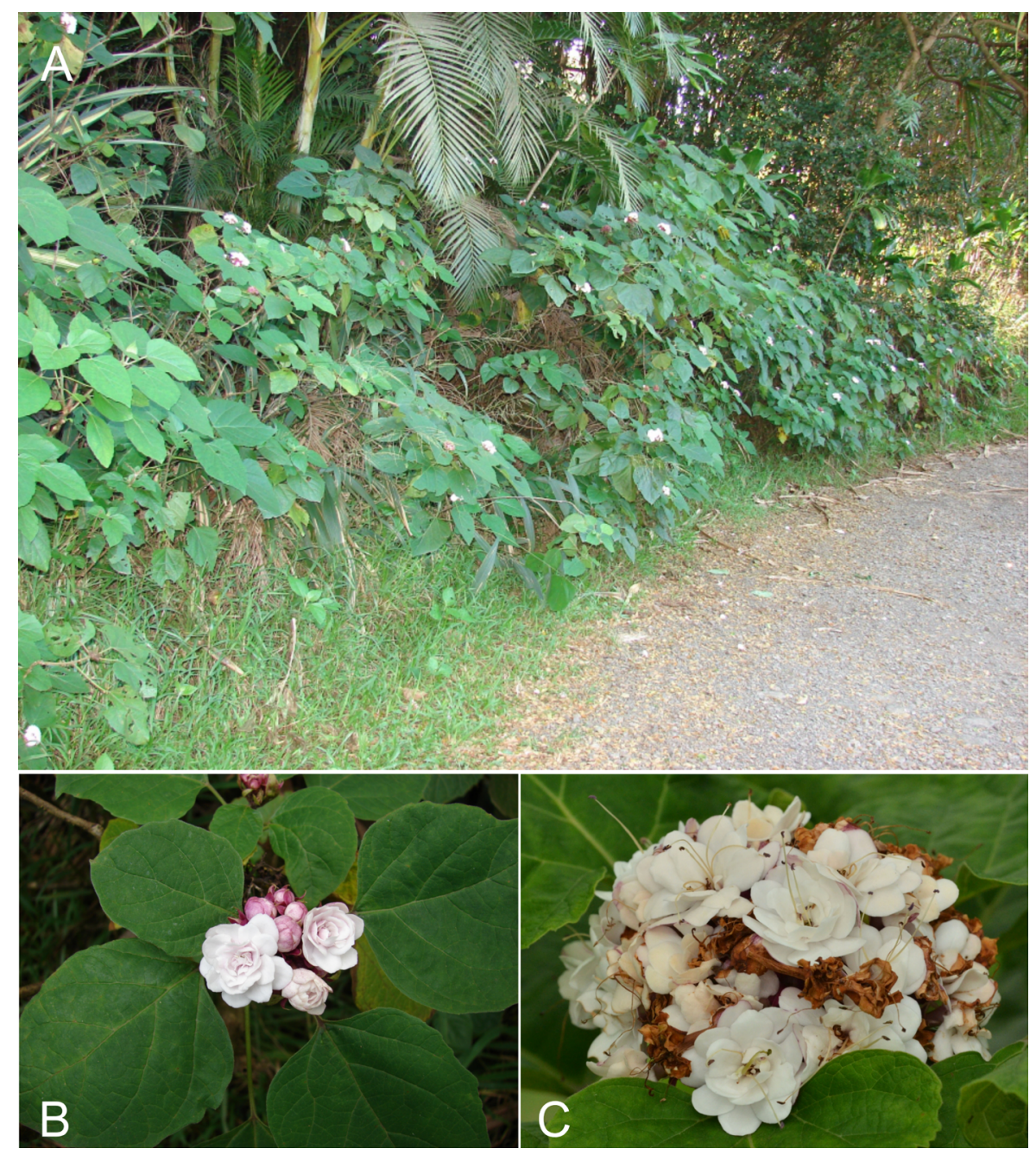

Fig. 2. Clerodendrum chinense. A: Hábito. B: Aspecto general de la inflorescencia y hojas. C: Detalle de la inflorescencia (Créditos fotográficos: A-C: Forest Starr \& Kim Starr).

Fig. 2. Clerodendrum chinense. A: Habit. B: General appearance of the inflorescence and leaves. C: Detail of the inflorescence (Photo credits: A-C: Forest Starr \& Kim Starr). 
de que el material colectado fuese cultivado. Por otro lado, la misma autora, pone en duda la procedencia del material de Venturi 286 (SI) para Tucumán, ya que afirma que la localidad en donde se coleccionó la especie, es una zona altamente antropizada, y que muy probablemente sea cultivada, agregando también, que el colector no indica en qué tipo de ambiente fue coleccionado dicho ejemplar. Sin embargo, en la etiqueta de herbario, como ocurre con el ejemplar colectado de $C$. bungei por el mismo botánico, se indica "terrenos incultos". Por este motivo, en vista de todas estas observaciones, consideramos que es muy probable que también esta especie se encuentre naturalizada en nuestro país, a pesar de no contar con nuevas colecciones para confirmar dicha presencia.
Material adicional estudiado: ARGENTINA. Entre Ríos: Dep. Villaguay, Villaguay, Est. Santa Martina, 1936-1937, fl, Müsch s.n. (SI-998). Misiones: Dep. Capital, Posadas, 23-X-1981, fl, Burkart 19741 (SI). Tucumán: Dep. Capital, Villa Luján, 460 m, III-1919, “terrenos incultos, a orillas del camino”, fl, Venturi 286 (SI).

Clerodendrum ekmanii Moldenke, Phytologia 1: 445. 1940. Fig. 3A-D

Distribución geográfica y ecología: Especie nativa del centro-oeste y sur del Brasil (Harley et al., 2015), centro-oeste de Paraguay y noreste de la Argentina; crece entre los 0-500 m (O’Leary, 2018; Zuloaga et al., 2019).

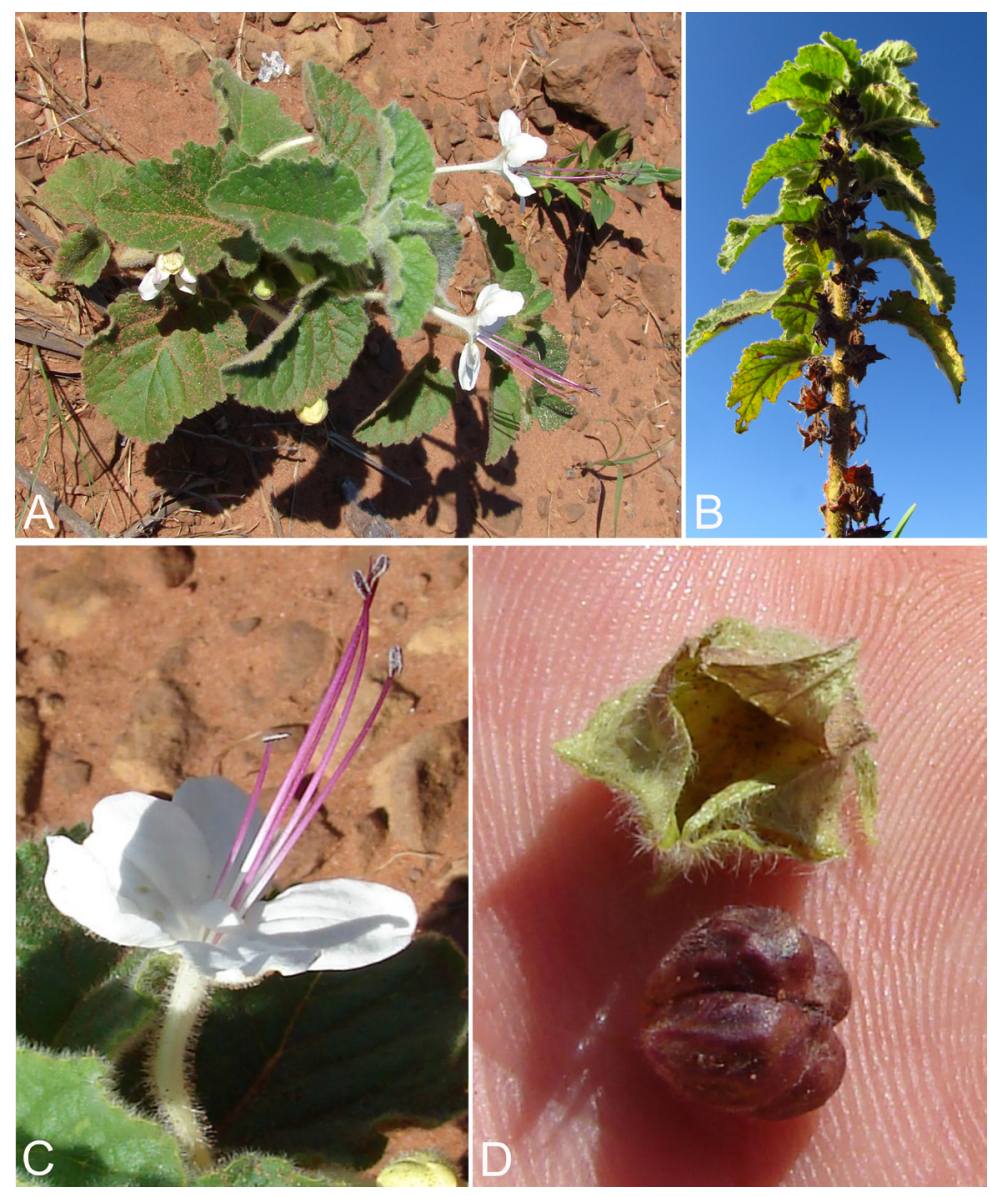

Fig. 3. Clerodendrum ekmanii. A: Hábito. B: Aspecto general, mostrando las inflorescencias axilares. C: Detalle de la flor. D: Cáliz (arriba) y fruto (abajo). (Créditos fotográficos: H. A. Keller).

Fig. 3. Clerodendrum ekmanii. A: Habit. B: General appearance, showing axillary inflorescences. C: Detail of the flower. D: Calyx (top) and fruit (bottom). (Photo credits: H. A. Keller). 
Materialadicionalestudiado:ARGENTINA. Misiones: Dep. San Ignacio, San Ignacio, 21-X1913, Quiroga s.n. (Holotypus, G00359487!).

\section{Agradecimientos}

Este estudio fue financiado en parte por la Coordenação de Aperfeiçoamento de Pessoal de Nível Superior-Brasil (CAPES). Un agradecimiento especial al Dr. Héctor Keller por las fotos de C. ekmanii y a Forest Starr \& Kim Starr por las fotos de $C$. chinense.

\section{Bibliografía}

Armitage, A. M. (2001). Armitage's manual of annuals, biennials, and half-hardy perennials. Portland, OR, USA: Timber Press.

Buchoski, M. G. (2021). Clerodendrum. En Flora do Brasil 2020 em construção. Jardim Botânico do Rio de Janeiro. Disponible: http://reflora.jbrj.gov.br/reflora/ floradobrasil/FB23325/ (Consulta: 04/01/2021).

Cantino, P. D. (1992). Evidence for a polyphyletic origin of the Labiatae. Annals Missouri Botanical Garden 79: 361-379.

Cantino, P. D., Harley, R. M. \& Wagstaff, S. J. (1992). Genera of Labiatae: status and classification. En Harley, E.M. \& T. REYNOLDS (eds.), Advances in Labiate Science, pp. 511-522. Royal Botanic Gardens, Kew.

Dimitri, M. J. (1980). Verbenaceae. En L. R. Parodi, Enciclopedia Argentina de Agricultura y Jardinería. Ed. Acme. Tomo 1: 924.

Flora Argentina. (2021). Instituto de Botánica Darwinion. Disponible: http://www.floraargentina. edu.ar (Consulta 16/01/2021).

Harley, R., França, F., Santos, E. P., Santos, J. S. \& Pastore, J. F. (2015). Lamiaceae. En Lista de Espécies da Flora do Brasil. Jardim Botânico do Rio de Janeiro. Disponible: http://floradobrasil.jbrj.gov.br/ jabot/floradobrasil/FB36510 (Consulta: 16/01/2021).

Hurrell, J. A. \& Delucchi, G. (2013). Aportes de la Etnobotánica al estudio de las invasiones biológicas. Casos en la región rioplatense (Argentina). Revista Historia Natural ( $3^{\mathrm{a}}$ serie) 3: 61-76.

Idárraga-Piedrahita, A., Ortiz, R. D. C., Callejas PosadA, R. \& Merello, M. (2011). Flora de Antioquia. Catálogo de las Plantas Vasculares, vol. 2. Listado de las Plantas Vasculares del Departamento de Antioquia.
Invasive Species Compendium. (2021). Detailed coverage of invasive species threatening livelihoods and the environment worldwide. Disponible: https://www.cabi. org/isc/datasheet/113370 (Consulta: 16/01/2021).

JARretT, A. (2003). Ornamental Tropical Shrubs, Pineapple Press Inc., Florida.

Jørgensen, P. M., Nee, M. H. \& Beck, S. G. (eds.) (2013). Catálogo de las plantas vasculares de Bolivia. Monographs in Systematic Botany from the Missouri Botanical Garden 127: 1-1741.

Мовот (2021). Gardening Help. St. Louis, MO, USA: Missouri Botanical Garden. Disponible: http://www. missouribotanicalgarden.org/PlantFinder/PlantFin derDetails. $87498 \&$ isprofile $=1 \&$ basic $=$ clerodendr um\%20bungei (Consulta: 16/01/2021).

MoldenKe, H. N. (1940). Novelties in the Avicenniaceae and Verbenaceae. Phytologia 1: 445-446.

Moldenke, H. N. (1948). The known geographic distribution of the members of the Verbenaceae, Avicenniaceae, Stilbaceae, and Symphoremaceae. Supplement 8, Castanea 13: 110-121.

Nesom, G. L. (2009). Assessment of invasiveness and ecological impact in non-native plants of Texas. Journal of the Botanical Research Institute of Texas 3: 971-991.

O'LeARY, N. (2018). Lamiales: Lamiaceae, Linderniaceae, Orobanchaceae, Plantaginaceae, Scrophulariaceae (coord. \& autor). En F. ZuloagA \& A. Anton (eds.), Flora Argentina 20: 1-488.

PAPF. (2021). Plants For A Future. Disponible: https://pfaf. org/user/Plant.aspx? LatinName $=$ Clerodendrum + bun gei (Consulta: 16/01/2021).

RAE (2021). REAL ACADEMIA ESPAÑOLA: Diccionario de la lengua española, 23. ${ }^{\text {a }}$ ed., [versión 23.4 en línea]. Disponible: https://dle.rae.es (Consulta: 02/03/2021).

RuedA, R. M. (1993). The genus Clerodendrum (Verbenaceae) in Mesoamerica. Annals of the Missouri Botanical Garden 80: 870-890.

Steane, D. A., KoK, R. P. J. DE \& Olmstead, R. G. (2004). Phylogenetic relationships between Clerodendrum (Lamiaceae) and other Ajugoid genera inferred from nuclear and chloroplast DNA sequence data. Molecular Phylogenetics and Evolution 32: 39-45.

ThIERs, B. (2021). Index Herbariorum: a global directory of public herbaria and associated staff. New York Botanical Garden's Virtual Herbarium. Disponible: http://sweetgum.nybg.org/ih (Consulta: 16/01/2021).

Troncoso, N. (1974). Los géneros de Verbenáceas de Sudamérica extratropical (Argentina, Chile, Bolivia, Paraguay, Uruguay y Sur de Brasil). Darwiniana 18: 295-412. 
Waterhouse, D. F. (1993). Biological control: Pacific prospects - supplement 2. Canberra, Australia, ACIAR: 72-93.

Wearn, J. A. \& Mabberley, D. J. (2011a). Clerodendrum confusion - redefinition of, and new perspectives for, a large Labiate genus. Gardens' Bulletin Singapore 63: 119-124.

Wearn, J. A. \& Mabberley, D. J. (2011b). Clerodendrum (Lamiaceae) in Borneo. Systematic Botany 36: 1050-1061.

Woodson, R. E., Schery, J. R. W. \& Moldenke, H. R. (1973). Flora of Panama. Part IX. Family 168. Verbenaceae. Annals of the Missouri Botanical Garden 60: 41-148.
Yuan, Y. W., Mabberley, D. J., Steane, D. A. \& Olmstead, R. G. (2010). Further disintegration and redefinition of Clerodendrum (Lamiaceae): implications for the understanding of the evolution of an intriguing breeding strategy. Taxon 59: 125-133.

Zuloaga, F. O., Morrone, O. \& Belgrano, M. J. (2008). (eds.). Catálogo de las Plantas Vasculares del Cono Sur (Argentina, sur de Brasil, Chile, Paraguay y Uruguay). Monographs in Systematic Botany from the Missouri Botanical Garden 107: 1-3348.

Zuloaga, F. O., Belgrano, M. J. \& Zanotti, C. A. (2019). Actualización del Catálogo de las Plantas Vasculares del Cono Sur. Darwiniana, nueva serie 7: 208-278. http://dx.doi.org/10.14522/darwiniana.2019.72.861 